\title{
A Research Program
}

on

\section{Radiative Transfer Model Development in Support of the ARM Program}

\author{
A research program funded by the \\ Office of Energy Research
}

Grant No. DE-FG02-90ER61064

For the period

1 March 1991 - 1 April 1992

Submitted by

Atmospheric and Environmental Research, Inc.

840 Memorial Drive

Cambridge, MA 02139

1 May 1992

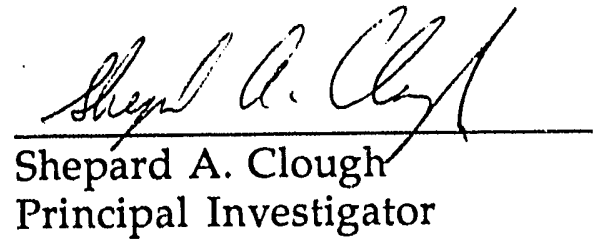

Principal Investigator

\section{DISCLAIMER}

This report was prepared as an account of work sponsored by an agency of the United States Government. Neither the United States Government nor any agency thereof, nor any of their bility for the accuracy, warranty, express or implied, or assumes any legal liability or responsibility for the accuracy, completeness, or usefulness of any information, apparatus, product, or process disclosed, or represents that its use would not infringe privately owned rights. Refermanufacturer, or otherwise does not necessact, process, or service by trade name, tradeinark, mendation, or favoring by the United necessarily constitute or imply its endorsement, rtcomand opinions of authors expressed herein do Government or any agency thereof. The views United States Government or any agency thereof. necessarily state or reflect those of the 


\section{Progress for the Reporting Period}

\subsection{Revised Continuum Model}

The water vapor continuum plays an important role in atmospheric radiative transfer providing increased opacity between spectral lines over the full spectral region from the microwave to the visible. The continuum has a significant influence on atmospheric fluxes and cooling rates. Additionally the continuum is important to the physical solution of the inverse problem, the remote sensing of atmospheric state to retrieve temperature, water vapor, surface properties and other state parameters. There are two components to the continuum: the self-broadened continuum, dependent on the square of the partial pressure of water vapor, and the foreign-broadened continuum, principally dependent on the product of the water vapor partial pressure and the total pressure. As a consequence the self broadened continuum tends to be more important in the lower atmosphere while the foreign broadened continuum tends to be more important in the mid to upper troposphere. A comprehensive continuum model based on a single line shape for all transitions from the microwave to the visible has provided generally acceptable results (Clough et al., 1989 and Clough et al., 1980; hereafter CKD). The nadir and zenith spectral radiometric measurements with the University of Wisconsin HIS instrument (Smith et al., 1983) have been particularly useful in establishing the general level of accuracy for the continuum. For most spectral regions the apparent error is of the order of $10 \%$ or less. The term 'apparent' is used because it is difficult to unambiguously characterize the atmosphere, particularly with respect to aerosol loading. However, for special conditions of atmospheric state and for very specific spectral region;, measurements indicate errors in the continuum may be as large as $60 \%$.

To address this situation and to improve overall accuracy, we have embarked on the development of an improved water vapor continuum modei. The full data set of Burch and coworkers $(1985,1984,1981)$ has been reanalyzed using a self-consistent approach for the contribution of local lines. In contrast to the earlier model, this reanalysis provides full consistency witl the line shape decomposition used for the FACADE algorithm. The present status of this work is that a new line shape is in the process of being 
transfer is used for the non-scattering atmosphere and provides the spectral boundary condition for the CHARTS calculation. The interface with the lineby-line model is currently being implemented.

A presentation on this work will be given by J.-L. Moncet at the IRC Symposium in Tallinn, Estonia in August, 1992. The related abstract is included.

\subsection{LBLRTM: Vectorized Line-by-Line Radiative Transfer Model}

Extensive effort has gone into two aspects of this code: (1) the acceleration of the model to facilitate real time spectral validations with ARM measurements and (2) the adaptation of ihe model to provide the necessary interface with CHARTS. LBLRTM is a vectorized line-by-line model based on the FASCODE algorithms and includes the capability to calculate atmospheric fluxes and heating rates, a capability developed under a related DoE grant, (No. DE-FG-02-86-ER60485). The modules performing these calculations have now been vectorized and are providing significantly accelerated performance on the CRAY-YMP. In the latter part of this year's grant we will perform a profiling analysis on the code to ascertain whether any significant improvements in performance are to be expected. The code has been passed through a 'FORTRAN Filter', resulting in increased readability and facilitating code analysis. LBLRTM does not include all the capabilities of FASCODE: the important limitations include the absence of the laser and two stream multiple scattering options. The availability of CHARTS addresses the latter issue. The Non-LTE option has not been validated in the new code and in particular has not been vectorized. A short document describing the capabilities and limitations of the model is available.

A second important capability included in LBLRTM is the provision to provide layer optical depths for CHARTS. The CHART calculations are performed at a monochromatic sampling interval determined by the line width of the scattering layer with the lowest pressure. LBLRTM provides the optical depths at this specified spectral spacing using a vectorized algorithm. The rninimum and maximum optical depths for the layer panels are included on the panel headers to facilitate the calculation of the doubling 
table for CHARTS. In addition to the more important issues described here a considerable number of bugs and minor problems have been addressed. Validations continue to be performed against HIS data.

Of related interest is the inclusion of LBLRTM as the forward model in the high resolution retrieval code that has been developed at AER. High resolution retrievals are computationally intensive if a line-by-line model is used for the forward problem. With the increased computational speed of LBLRTM this problem is somewhat alleviated for vectorizing platforms. For the inversion problem, the Jacobian of the forward model with respect to the state parameters to be optimized is required. It turns out that portions of the task of obtaining the Jacobian can be done analytically and quite straightforwardly in the context of the current development. Research in this direction is being pursued at a moderately low level for a number of related research efforts. In the near future a version of LBLRTM can be expected to rapidly provide derivatives of the spectral radiances with respect to atmospheric state parameters.

In related vein, we are exploring the feasibility of using an array processor in conjunction with a RISC based workstation for LBLRTM, CHARTS and related retrieval models. If this platform proves successful, a platform would be available at relatively modest cost to address these types of calculations for ARM applications. The cost of the array processor is of the order of $\$ 10,000-\$ 15,000$. As an initial step we are compiling the code with a vendor supplied compiler on an AER platform, a SPARC II workstation. If this step is successful we will either have the vendor benchmark the code in their environment or accept their offer to implement the array processor on our workstation on a trial basis.

\subsection{Rapid Model}

The main effort on improvements on the rapid model have focussed on the incorporation of the CKD continuum into the model and on the difficult problem of using the correlated-k method for spectral regions that have contributions from non-uniformly mixed gases (i.e. water vapol) in the presence of contributions from uniformly mixed gases (i.e. carbon dioxide). 
This is an old problem that has been addressed in a number of studies (Lacis and Oinas, 1991; Goody et al., 1989). We are pursuing some directions that we hope will prove fruitful but there is a real sense in which a fully physical treatment of this problem may not be possible and one will have to rely on parameterized corrections. We have developed optical depths through the tropopause for the spectral region from 590-645 $\mathrm{cm}^{-1}$ using LBLRTM for water vapor and carbon dioxide, both separately and combined, which are being used to study the problem in detail.

\subsection{Code Maintenance and Availability}

A problem that has arisen and remains unresolved relates to the archiving and maintaining of code. At present we are supplying codes to a number of ARM science team members which has necessitated the porting of code to their environment. Operating in this fashion, it is difficult given our interests and our resources, to provide a proper structure for maintaining the codes. It had been anticipated that ARM would provide a repository site for code archival but given the extent of current demands, that evidently is not feasible. We are exploring the possibility of providing this capability at AER for AER developed code. ARM Science team members would be able to access a designated ftp account. Although our present thought is that this would include only code developed at AER, a broader context could be considered. The main requirement for AER is that sufficient disk space be available on the special ftp account and that there not be an adverse impact on the AER network.

In addition to AER, LBLRTM has been implemented at Florida State Unirersity (cray-ymp), Pacific Northwest Laboratories (Convex and Cray), the Pennsylvania State University (cray-ymp), the Jet Propulsion Laboratory (crayymp) and at the U. of Maryland (RISC based workstation). The versions implemented at these sites have some differences dependent on the time of implementation. As of June 1992 these codes will be available with SCCS revision numbers and the code will be maintained consistent with that archiving system. The $\mathrm{X}$-window interface has been implemented and is operational at Penn. State U. 


\subsection{Real Time Model/Measurement Validation}

The question has arisen in terms of our ARM related research as to what spectral data we would like to receive and how we would use that data. The central point in responding to this question is that our project does not have the resources nor the interest of the scientists involved to ingest and analyze large amounts of data. Our interest in validating and improving the capability of spectral radiative models would best be served by having a zeroth order analysis of all the spectral data performed in real time, evaluate the comparison against established criteria for unsatisfactory model or data performance and make available those data sets with unsatisfactory validations for further study. It is our understanding that this approach involves certain complications which we hope can be alleviated. Jim Liljegren has provided a draft document on implementing this approach and we anticipate working with him and other interested science team members to proceed as far as possible in this direction. We do feel that looking at all the data is extremely important since 'anomalous' events have often turned out to be the most interesting and could easily be missed by a data sampling approach. 


\section{Presentations}

1. A review of our work under the ARM program was presented at the Scientific Team Meeting in Denver, 1991. Much of the work described here is included in the extended abstract provided for that meeting.

2. This research formed the substance of material presented at the Pennsylvania State University Meteorology Symposium by S. A. Clough in September 1991.

\section{Travel}

J.-L. Moncet and S.A. Clough traveled to Pennsylvania State University to meet with Tom Ackerman and present a colloquium, September 1991.

S. A. Clough traveled to Denver, $\mathrm{CO}$ to attend the ARM science team meeting, December 1991.

Partial support has been utilized to attend the NATO conference on high resolution measurements from space for remote sensing and climate studies, March 1992. A paper on validations with the HIS measurements on and the water vapor continuum was presented. 
Ma, Q., and R. H. Tipping, A far wing line shape theory and its application to the water continuum absorption in the infrared region (I), I. Chem. Phys. $\underline{X X}$ xxxX-XXXX, 1992a.

Ma, Q., and R. H. Tipping, A far wing line shape theory and its application to the water continuum absorption in the infrared region (II), I. Chem. Phys. $\underline{X X}, \underline{x x X-X x x x, 1992 b .}$

Roberts, R. E., J. E. A. Selby, and L. M. Biberman, Infrared continuum absorption by atmospheric water vapor in the 8-12 $\mathrm{mm}$ window, Appl. Opt. 15, 2085-2090, 1976.

Smith, W. L., H. E. Revercomb, H. B. Howell, and H. M. Woolf, HIS - A satellite instrument to observe temperature and humidity profiles with vertical resolution, Proc. Fifth Conference on Atmospheric Radiation, AMS, 1983. 


\section{1 \\ $r$}

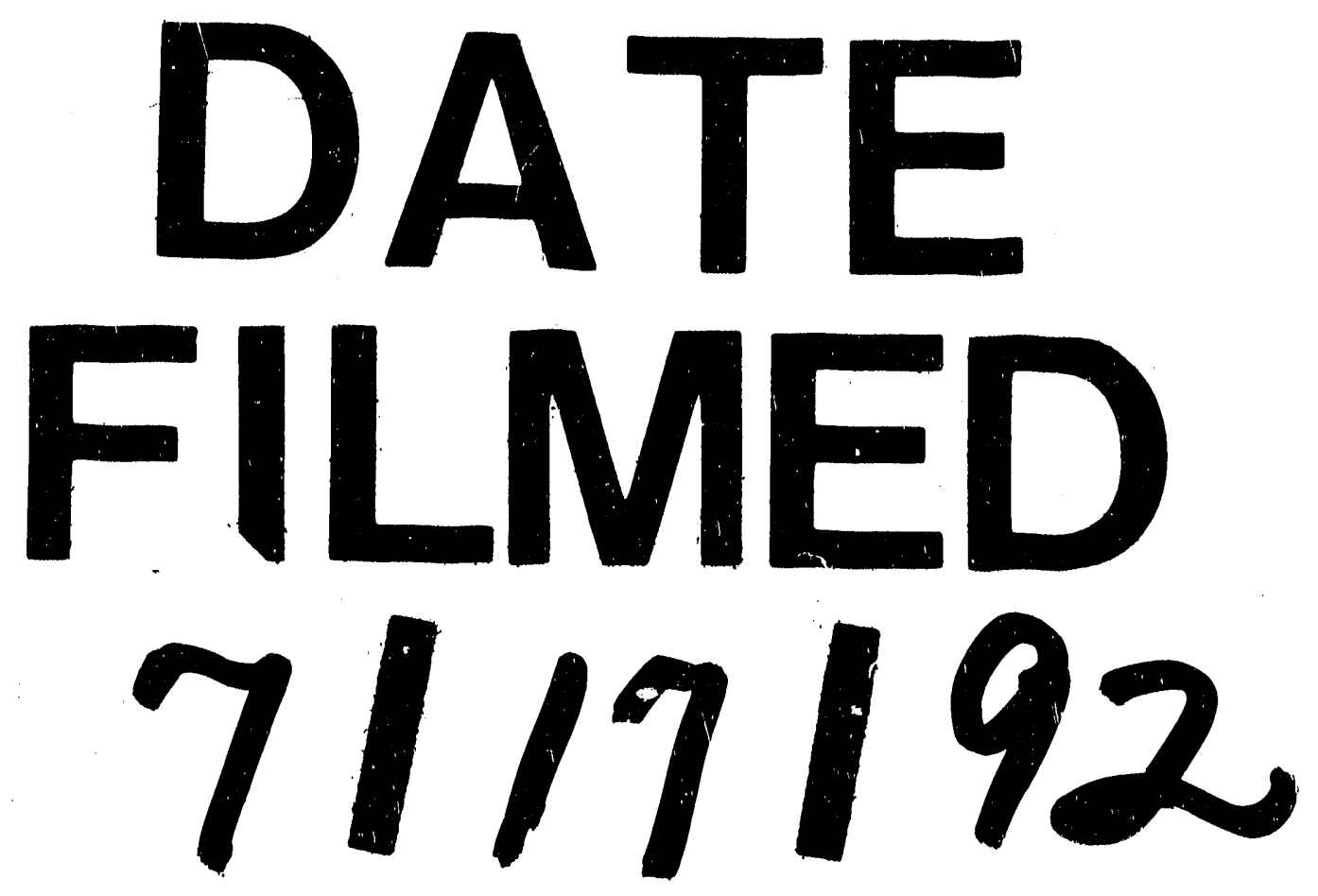




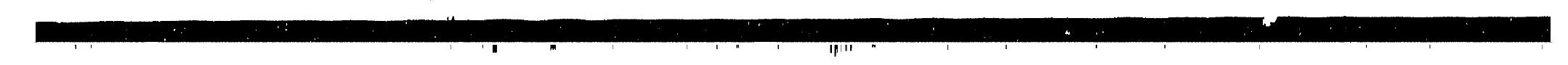

